\title{
Propagation of Cosmic Rays from the Vicinity of the Galactic Centre
}

\author{
R. W. Clay \\ Department of Physics and Mathematical Physics, \\ University of Adelaide, SA 5005, Australia \\ rclay@physics.adelaide.edu.au \\ Received 2000 February 2, accepted 2000 June 6
}

\begin{abstract}
There is now evidence that there may be a strong source of cosmic ray particles in the general direction of the Galactic Centre. The likelihood is that the observed particles are neutrons with energies of about $10^{18} \mathrm{eV}$. Associated with the production of those neutrons, we would expect that large numbers of charged cosmic rays would also be produced, and we investigate here the directional properties of those charged particles as they may be observed at the distance of the Earth from the Galactic Centre. We follow the propagation of such particles through a simple Galactic magnetic field model with both a turbulent and a regular field to determine what field properties most affect the observed beam. It appears that the turbulent field component is crucial to any resulting charged particle observations.
\end{abstract}

Keywords: cosmic rays-ISM: magnetic fields

\section{Introduction}

Recent results from the AGASA cosmic ray air shower array in Japan (Hayashida et al. 1999a, 1999b) have suggested that there is a source of ultra-high-energy cosmic rays in the general direction of the Galactic Centre. Those results were highly suggestive of a Galactic Centre source with an excess of cosmic ray intensity of about $25 \%$ from a region in the Galactic Plane about $10^{\circ}$ from the Centre. This compact source contributed to the broad-scale $4 \%$ first harmonic of the cosmic ray anisotropy reported by Hayashida et al. A region of lesser intensity extends for a few tens of degrees northwards into the AGASA field of view, and there is a less convincing enhancement in the Cygnus region of the Galactic Plane, close to the direction of the inward spiral arm. The high latitude of the array and the southern declination of the Galactic Centre meant that the Centre itself was just outside the view of the array and it was not clear whether the main excess region extended to the Galactic Centre direction.

The major strength of the source signal was at energies a little over $10^{18} \mathrm{eV}$ and was restricted to a range of energies of about a factor of three. This meant that the only other array capable of observing the source, SUGAR (the Sydney University Giant Air Shower Recorder), had not detected the source due to its statistically poorer dataset. Knowing that a signal might exist, Clay et al. (2000) were able to confirm the observation with the SUGAR dataset. Further detailed examination of the SUGAR data (Bellido, Clay \& Dawson 2000) found that the source appeared to be pointlike (and coincident with the strongest AGASA direction) and did not extend to the direction of the Galactic Centre.

A simple explanation of a pointlike source of cosmic rays is that they are neutral particles. The fact that the source is observed only close to $10^{18} \mathrm{eV}$ is strongly suggestive that they are neutrons, which have a lifetime just sufficient to reach us from the distance of the Galactic Centre at those energies. At lower energies, the effect of time dilation is less and lower-energy neutrons would not survive to produce a point source. One expects Galactic cosmic ray acceleration to have an energetic upper limit not much greater than about $10^{18}$ $\mathrm{eV}$, so the limited range of observed energies is naturally explained (Clay et al. 2000).

One presumes that the neutrons are produced by the conversion of accelerated particles in a target close to the source and so the question of the propagation of the non-converted particles becomes of interest. Jones (1990) has discussed the production of neutrons at energies such as this. In the case of neutron production by both inelastic charge exchange of protons and the dissociation of primary nuclei, there are more neutrons produced than gamma-rays. If the source emits nuclei together with protons, their propagation paths will be scattered more than for the protons in the discussion below, and they would be unlikely to be identifiable over the more general cosmic ray background.

We have modelled the propagation of cosmic ray protons from the general vicinity of the Galactic Centre (as explained above, this would seem to be the appropriate distance and to be roughly the right direction) to the distance $(8.5 \mathrm{kpc})$ of the Earth through a simple model of the Galactic magnetic field.

\section{Propagation Modelling and the Magnetic Fields}

When Galactic cosmic ray trajectory calculations have been made in the past (e.g. Lee \& Clay 1995), they have often been based on the reverse propagation of antiprotons from the Earth. This allows one to follow the path of incoming particles and to determine their pathlengths within the Galaxy as a function of arrival direction. In the case of a source at the Galactic Centre, this procedure is not possible as both a particular source and an end point are specified. For this procedure to be 
practical, we have used the Galactic Centre as the source of protons and accepted particles which reach the Galactocentric distance of the Earth over a range of distances above or below the Galactic Plane. That distance was chosen to be $1 \mathrm{kpc}$. The azimuthal symmetry of our assumed field allows us to use any particle reaching that distance from the Centre.

In this work, we followed Lee \& Clay (1995) and adopted the axisymmetric Galactic magnetic field model of Rand \& Kulkarni (1989). This models the spiral arms as concentric rings. The rings correspond to the distances from us of the Galactic spiral arms, and the magnetic field is assumed to be in azimuthal directions. In this case, we assume that the Earth is at a distance of $8.5 \mathrm{kpc}$ from the Galactic Centre and that the centres of the spiral arm 'rings' are at distances of $9.4 \mathrm{kpc}$ and 3.4 $\mathrm{kpc}$ from the Centre for one field polarity and at $6.4 \mathrm{kpc}$ for the reverse polarity. The field strength in the rings is assumed to vary sinusoidally with distance from the Galactic Centre with an amplitude of $2.15 \mu \mathrm{G}$. Locally, the field is then towards a Galactic longitude of $90^{\circ}$. Following Lee \& Clay, we have included a random magnetic field component at a similar magnitude to that which is observed locally, with the assumption that the magnitude of the random field is proportional to the magnitude of the local regular field. We have followed protons of different energies through fields of different strengths with various relative strengths of the regular and random components.

Compared to Lee and Clay, we assume a rather weaker dependence of the field on distance above and below the Galactic Plane. We assumed an e-folding distance with $z$ of 500 pc rather than 100 pc. This doubled the number of events available in our resulting dataset. Additionally, we placed the Earth at a Galactocentric distance of $8.5 \mathrm{kpc}$ rather than the previous 10 $\mathrm{kpc}$. This is rather closer to present convention. The regular field strength varies sinusoidally with distance from the Galactic Centre, with a spatial period of $6 \mathrm{kpc}$ to simulate both the effect of increased field strength in the spiral arms and the reversal from one arm to the next. A random magnetic field was added to make up the complete field. This turbulent field had a Kolmogorov spectrum and a maximum scale size of 100 pc (see Lee \& Clay 1995). The field strength associated with the maximum scale size was 2.5 times that of the regular field.

Cosmic ray protons were launched from the position of the Galactic Centre. They were assumed to be lost from the Galaxy if their distance above or below the Plane exceeded $1 \mathrm{kpc}$. For this reason, their initial elevations were chosen randomly from a solid-angle distribution limited to within $30^{\circ}$ of the Galactic Plane. Experience showed that particles with elevations outside this range were soon lost from the Galaxy. The protons were followed until they left the Galaxy at a distance of $1 \mathrm{kpc}$ above or below the Galactic Plane, or reached a distance of $8.5 \mathrm{kpc}$ from the Centre, thus reaching the
'Earth' target. As noted above, the choice of $1 \mathrm{kpc}$ was dictated by the need to retain a significant number of events. The distribution of final distances above or below the Plane was, however, broad.

On reaching the distance of the 'Earth', the position of the proton was recorded together with its current velocity vector, the reverse of which would correspond to the direction of its apparent source as recorded by a cosmic ray detector.

\section{Beam Properties at the Distance of the Earth}

As a check on the properties of propagation in the turbulent field, we ran the program with a purely random field. Clay et al. (1998) have previously calculated the properties of proton propagation in turbulent intergalactic fields and, apart from energy loss issues as discussed by Clay et al. (1998, 2000) and details of the field strength, their broad results should offer a check on our propagation. We find that, as expected, the mean angular deviation of the observed particles from the direction of the Galactic Centre is inversely proportional to energy for small angular spreads. At $10^{19} \mathrm{eV}$ it has a value of $6^{\circ}$, fortuitously almost identical to that expected on the basis of the previous work and its simple argument concerning energy losses. At $10^{18} \mathrm{eV}$, a decade lower in energy, the propagation is close to diffusive with an almost complete loss of directionality.

When the regular field is introduced, a shift of the mean direction of the observed particles from the direction of the Galactic Plane results. The magnitude of this deviation depends on the energy of the particles but the dependence is not simple. For our assumed regular magnetic field, we find an overall deflection of the observation direction to the north from the Galactic Plane. This is to be expected for the local field direction used in the model. However, the magnitude of the deflection is not now inversely proportional to energy except, perhaps, above $10^{19} \mathrm{eV}$.

There are at least two contributing factors to this complexity. As we have just noted, the deflection due to the random field is significant at energies appreciably below $10^{19} \mathrm{eV}$, and in such a situation one does not expect linearity to apply to the mean deflection. Also, the spatial period of the regular field as viewed by a particle travelling from the Galactic Centre is of the order of the gyroradius of a particle at these energies. We might well expect that the propagation would show systematic changes in character with energy but it is unreasonable to expect a simple relationship so close to a resonant situation. At $10^{19} \mathrm{eV}$, the systematic deflection north of the direction of the Galactic Plane is of the order of $10^{\circ}$.

The complexity of the dependence of the propagation on local conditions is emphasised if one considers potential observations at different positions within the spiral arm magnetic field. The Earth is towards the inside edge of our spiral arm. Propagation to us is therefore influenced by both our spiral arm field and the field 
of the next arm inwards, which is in the reverse direction. This emphasises that the detailed properties of the propagation, and the resulting observed arrival directions, depend rather critically on knowing the correct placement of the observation point within the Galactic magnetic field.

In reaching the distance of the Earth from the Galactic Centre, a particle must travel through systematic fields which tend to deflect it away from the Galactic Plane. It is clear that many particles which have gyroradii smaller than the Galactocentric distance of the Earth are unlikely to reach us without some assistance. That assistance may well come from the turbulent field. Using our simple magnetic field model as an example, if we take protons with an energy of $5 \times 10^{18} \mathrm{eV}$ and investigate the situation $1 \mathrm{kpc}$ more distant than us, the number of observed particles per particle emitted from the Galactic Centre increases by over 20 times for a factor of 4 increase in the strength of the random field. At the position of the Earth, there is a similar, but weaker, effect. Clearly, the random field can have the effect of returning some wayward particles back towards the direction of the Galactic Plane. Reversing the argument, one could also note that a regular field of value greater than only about 0.03 times that of the random field is sufficient to produce an observable reduction in the detection rate when compared to a purely random field.

In general, it is very difficult for a particle to reach the Galactocentric distance of the Earth if its energy is below about $3 \times 10^{18} \mathrm{eV}$, with this threshold depending on the detail of the assumed field. With our best model of the field and the position of the Earth, about one particle in one thousand reached the Galactocentric distance of the Earth at $2.5 \times 10^{18} \mathrm{eV}$. If the Earth had been assumed to be close to the centre of our spiral arm (i.e. a little more distant then we have assumed so far), this would fall to below one in ten thousand. At $10^{19} \mathrm{eV}$, the success rate increased to about one in five for our best model, approaching the value to be expected on solidangle arguments for the targeted band of distances from the Galactic Plane at the distance of the Galactic Centre.

\section{Conclusions}

A number of aspects of these calculations are relevant to the interpretation of observational data concerning charged particles from a potential Galactic Centre source. The spread of the arrival directions is of interest, as is the mean value of the arrival direction north of the Galactic Plane. Both of these are of the order of a few degrees at $10^{19} \mathrm{eV}$ and reduce with increasing energy. We would also like to have an idea of how likely it is that a particle might reach the observational region. This is very unlikely below $10^{18} \mathrm{eV}$ but reaches a value expected on the basis of solid-angle geometry at about $10^{19} \mathrm{eV}$ where the propagation is becoming rather rectilinear. The way in which the propagation changes between these energies depends on detail of the magnetic field that is presently unknown. In this range of energies, it is clear that the interplay between the Galaxy's regular field and its random field is crucial in the propagation of the particles and thus their likelihood of reaching us and being observed in a particular direction. With the inclusion of a regular field, there is a systematic deviation of the observation direction to the north, based on our best knowledge of the true field.

Thus, for quite different reasons, it appears that a source in the vicinity of the Galactic Centre would be unobservable below $10^{18} \mathrm{eV}$ with either charged particles or neutrons. At energies above this, the neutrons would produce a point source and the charged particles (protons) would produce a halo of energy-dependent size and location, north of the Galactic Plane. In previous work it has been noted that charged particles can become attached to the regular spiral arm field lines, and we would additionally expect some of the charged particles to be deflected along the spiral arm, producing an excess in the general direction of its magnetic field.

\section{References}

Bellido, J. A., Clay, R. W., \& Dawson, B. R. 2000, Astroparticle Phys., in press

Clay, R. W., et al. 1998, Astroparticle Phys., 9, 221

Clay, R. W., et al. 2000, Astroparticle Phys., 12, 249

Hayashida, N., et al. 1999a, Astroparticle Phys., 10, 303

Hayashida, N., et al. 1999b, Proc. 26th Int. Cosmic Ray Conf. (Salt Lake City) 3, 256

Jones, L. W. 1990, Proc. 21st Int. Cosmic Ray Conf. (Adelaide) 2, 75

Lee, A. A., \& Clay, R. W. 1995, J. Phys. G: Nucl. Part. Phys., 21, 1743

Rand, R. J., \& Kulkarni, S. R. 1989, ApJ, 343, 760 